\title{
AVALIAÇÃO DOS EFEITOS ALELOPÁTICOS DE DIFERENTES TIPOS DE SOLO NA GERMINAÇÃO DE ALFACE
}

\begin{abstract}
Josimar Rodrigues Oliveira - Graduando em Engenharia Agronômica e Bolsista do CNPq, Instituto
Federal de Educação, Ciência e Tecnologia Minas Gerais (IFMG) - Campus Bambuí, josimarodrigues@yahoo.com.br.D.Sc.Neimar de Freitas Duarte - IFMG - Campus Bambui, neimar@ifmg.edu.br. Breno Oliveira Rodrigues - Graduando em Engenharia Agronômica, IFMG Campus Bambui, brenoagronomia@yahoo.com.br

Henrique José G. M. Maluf-Graduando em Engenharia Agronômica, IFMG - Campus Bambuí, rick.maluf@yahoo.com.br. Isaac Alves Tonaco - Graduando em Engenharia Agronômica, IFMG Campus Bambui, isaacalvestonaco@yahoo.com.br. Matheus Silva Luiz - Graduando em Engenharia Agronômica, IFMG - Campus Bambuí, matheussilvaluiz@yahoo.com.br
\end{abstract}

\section{RESUMO}

O objetivo deste trabalho foi avaliar a influência de três diferentes solos superficiais na germinação e desenvolvimento de alface. Foi utilizado delineamento experimental inteiramente casualizados (DIC), com três tratamentos e quatro repetições. Os tratamentos são: T1 - Solo superficial de eucalyptus urophylla, T2 - Solo superficial de eucalyptus camaldulensis e T3 - Solo superficial de Latossolo vermelho de pastagem. Realizou-se a semeadura direta nos recipientes, colocando-se três sementes de alface Regina (Horticeres Sementes), com 98\% de taxa de germinação, tratadas com 0,15\% de Captan. Os recipientes foram colocados em ambiente com luminosidade controlada, utilizando-se sombrete $50 \%$. Após a germinação, as plântulas foram desbastadas deixando somente uma planta central por recipiente. Aos 24 dias após o plantio, foram mensurados o número de folhas, desconsiderando-se apenas os pares cotiledonares na contagem. Os tratamentos apresentaram diferenças estatisticamente significativas entre si, porém a alface apresentou melhor desenvolvimento nos tratamento com solo superficial de eucalyptus camaldulensis e eucalyptus urophilla, que não apresentaram efeito alelopático na cultura, porém no tratamento com solo superficial de um Latossolo Vermelho cultivado com pastagem, a cultura apresentou um desenvolvimento muito inferior quando comparado às médias dos demais tratamentos.

Palavras-Chave: Alelopatia, Lactuca sativa, Eucalyptus, Brachiaria sp.

\section{Evaluation of the allelopathics effects of diferents soils in germination of the lettuce}

\section{ABSTRACT}

The objective of this work was to evaluate the influence of three different superficial soils in the germination and development of lettuce. Experimental delineation was used entirely randomized, with three treatments and four repetitions. The treatments are: T1-Soil superficial of Eucalyptus urophylla, T2 - Soil superficial of Eucalyptus camaldulensis and T3 - Soil superficial of red Latossolo of pasture. It was become fullfilled direct sowing in the containers, placing itself three seeds of Regina lettuce (Horticeres Seeds), with $98 \%$ of germination tax, treated with $0,15 \%$ to Captan. The containers had been placed in environment with controlled luminosity, using sombrete $50 \%$. After the germination, seedlings had been roughed-hew only leaving a central plant for container. To the 24 days after the plantation, had been measured the leaf number, disrespecting itself only the pairs of follicles in the counting. The treatments had presented significantly differences between itself statistical, however the 
lettuce more good presented development in the treatment with ground superficial of Eucalyptus camaldulensis and Eucalyptus urophilla, that they had not presented allelopathic effect in the culture, however in the superficial treatment with ground of a cultivated Red Latossolo with pasture, the culture very presented an inferior development when compared with the averages of the too much treatments.

Key-Words: Allelopaty, Lactuca sativa, Eucalyptus, Brachiaria sp.

\section{INTRODUÇÃO}

A alelopatia pode ser definida como a interferência positiva ou negativa de compostos metabólicos secundários produzidos por uma planta ou microorganismos e lançados no meio atuando sobre o desenvolvimento de outra planta (MOLISCH, 1937; RICE, 1984; FERREIRA e BORGHETTI, 2004). Geralmente, ocorre alteração no padrão e na densidade, sendo um fenômeno comum que ocorre largamente em comunidades de plantas.

Severino et. al (2007), afirmam que a alelopatia não se caracteriza como uma competição, pois não ocorre uma disputa de recursos limitados como luz, água, nutrientes e espaço físico. A alelopatia trata-se de um efeito tóxico de substâncias produzidas por outras plantas.

A alelopatia pode ser uma estratégia ecológica de competição, pois através desse mecanismo, uma planta pode interferir no crescimento da outra, reconhecida como um importante mecanismo ecológico que influencia a dominância vegetal, a sucessão, a formação de comunidades vegetais e de vegetação clímax, bem como a produtividade e manejo de culturas.

Algumas leguminosas, gramíneas e hortaliças são comumente utilizadas em ensaios de alelopatia, uma vez que são de fácil estudo e muitas das vezes são implantadas em áreas próximas a florestas de Eucalipto ou em áreas anteriormente cultivadas com esta espécie.

Uma das hortaliças mais comum é alface.
Segundo Figueira (2005), caracteriza-se por ser uma planta herbácea, muito delicada, com caule diminuto não ramificado. As folhas são muito grandes, lisas ou crespas, fechando-se ou não na forma de "cabeça", estando presas ao caule.

A principal vantagem do uso de Lactuca sativa como alvo nos estudos alelopáticos reside na sensibilidade da espécie, mesmo em baixas concentrações de aleloquímicos. Além disso, de acordo com Souza (2005), a espécie apresenta outras peculiaridades que favorecem sua utilização, como germinação rápida, crescimento linear insensível às diferenças de pH em ampla faixa de variação e insensibilidade aos potenciais osmóticos.

Visto a importância do estudo de plantas com possíveis efeitos alelopáticos é justificado pela necessidade do desenvolvimento de uma agricultura sustentável. O objetivo deste trabalho foi avaliar a influência de três diferentes solos superficiais (solo de eucalyptus urophylla, solo de eucalyptus camaldulensis, e Latossolo vermelho de pastagem) na germinação e desenvolvimento de alface em condições de campo.

\section{MATERIAL E MÉTODO}

Foi utilizado delineamento experimental inteiramente casualizados (DIC), com três tratamentos e quatro repetições. Os tratamentos são: T1 - Solo superficial de eucalyptus urophylla, T2 - Solo superficial de eucalyptus camaldulensis e T3 - Solo superficial de Latossolo vermelho de pastagem.

O solos foram coletados no Instituto Federal Minas Gerais - Campus Bambuí, próximo a Unidade Educativa de Produção Agricultura III, retirando-se manualmente a camada superficial próximo ao colo da planta. Realizou-se a desintegração e homogeneização do solo, acondicionando-os em recipientes de aproximadamente $1 \mathrm{dm}^{3}$.

A semeadura direta nos recipientes foi realizada no dia 10 de novembro de 2009 , 
colocando-se três sementes de alface Regina (Horticeres Sementes), com 98\% de taxa de germinação, tratadas com $0,15 \%$ de Captan. Os recipientes foram colocados em ambiente com luminosidade controlada, utilizando-se sombrete $50 \%$.

Não foram realizados nenhum tipo de correção química ou fertilização do solo coletado para o cultivo de alface. As irrigações eram feitas diariamente com aproximadamente 2 litros de água em todos os tratamentos.

Após a germinação, as plântulas foram desbastadas deixando somente uma planta central por recipiente. Aos 24 dias após o plantio, foram mensurados o número de folhas, desconsiderando-se apenas os pares cotiledonares na contagem.

Os dados foram submetidos à análise de variância e testes de comparações de médias, através do Teste de Tukey a 5\% de probabilidade, pelo programa estatístico SISVAR 5.0 (FERREIRA, 2007).

\section{RESULTADO E DISCUSSÃO}

A sementes de alface germinaram normalmente nos solos cultivados por $E$. urophylla e E. camaldulensis, já no Latossolo vermelho de pastagem a germinação foi retardada, possivelmente devido ao alto teor de argila deste solo, que se mostrou propício à compactação desde as primeiras irrigações (Figura 1).

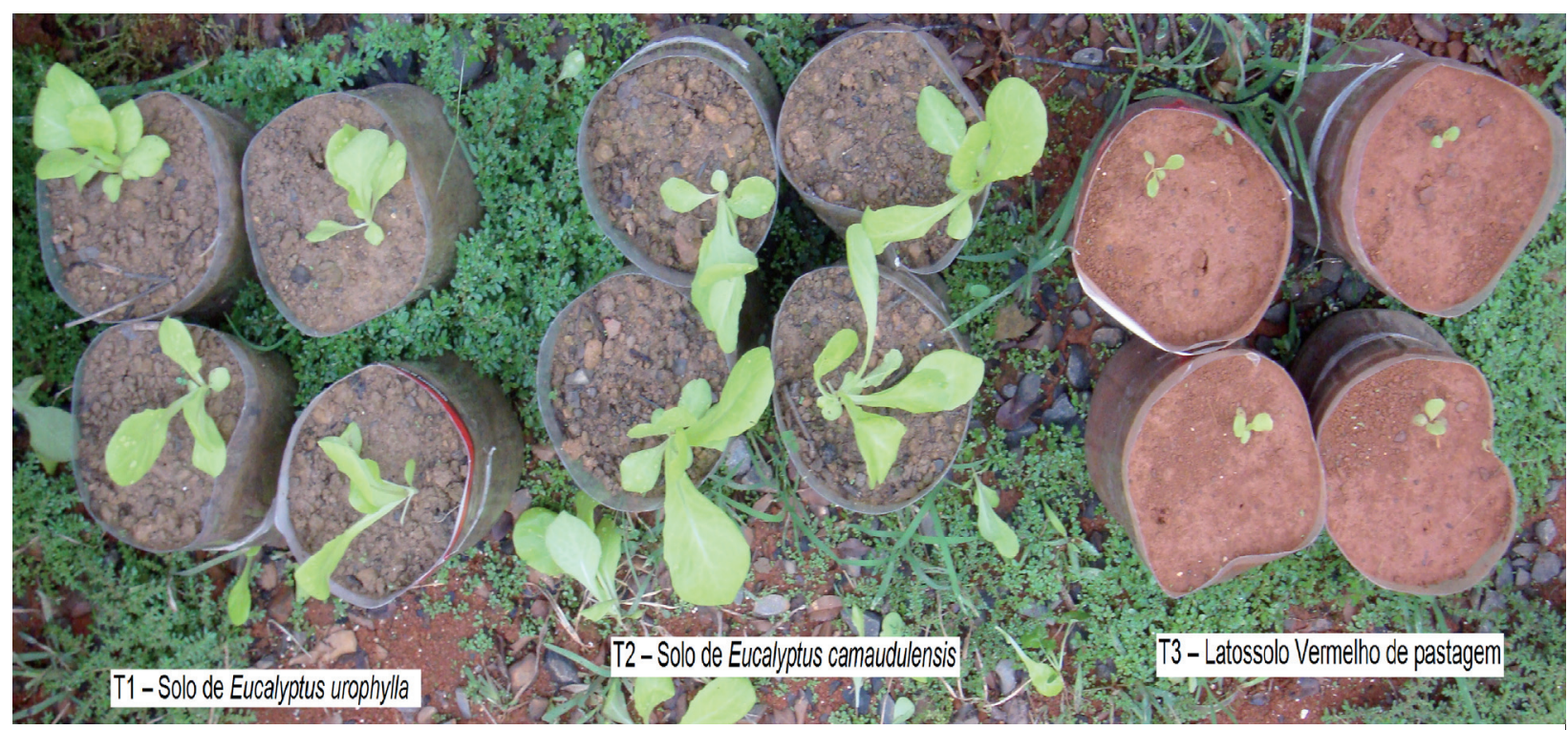

Figura 1 - Alface submetida a diferentes tratamentos

Na tabela 1 são apresentados os dados referentes à análise da variância, pode-se observar que entre os tratamentos houve diferenças estatisticamente significantes, podemos avaliar esta diferença analisando o nível do valor Pr, gerado pelos programas estatísticos em relação ao Fc (Valor de $\mathrm{F}$ calculado), se o valor Pr é menor que o nível de significância do teste, indica diferença estatística entre os tratamentos.

Este método equivale à comparação do Fc com o Ft (Valor F tabelado), neste caso, Pr equivale a 0,0222 para a fonte de variação (FV) tratamento, sendo menor que 0,05 (nível de significância do teste F), já no caso da repetição, não houve diferenças estatísticas entre si, pois Pr é $0,9263>0,05$; o que é desejável para um experimento. 
TABELA 1. Análise da Variância do Número de folhas de Lactuca sativa cultivada em diferentes solos.Bambuí, 2009.

\begin{tabular}{lrrrrr}
\hline \multicolumn{1}{c}{ FV } & GL & \multicolumn{1}{c}{ SQ } & QM & FC & Pr $>$ Fc \\
\hline & & & & & \\
\hline Tratamento & 2 & 54.167 & 27.083 & $7.677^{* *}$ & 0.0222 \\
Repetição & 3 & 1.583 & 0.528 & $0.150^{\text {ns }}$ & 0.9263 \\
Erro & 6 & 21.167 & 3.528 & & \\
\hline
\end{tabular}

\begin{tabular}{lrr}
\hline Total corrigido & 11 & 76.917 \\
\hline $\mathrm{CV}(\%)$ & 38.20 & \\
\hline
\end{tabular}

** Significativo ao nível de significância de 0,05 de probabilidade

$\mathrm{Na}$ avaliação do número de folhas foi observadoqueparaasplantassemeadas diretamente sobre solo superficial cultivado com E. urophylla (T1), obteve-se boa taxa de germinação, com desenvolvimento estatisticamente semelhante aos demais tratamentos, porém sobressaindo-se com média de seis folhas totalmente formadas aos 24 dias após o plantio (Figura 2).

Já as alfaces semeadas em solo superficial cultivado com E. camaldulensis (T2), foi estatisticamente diferente em relação ao solo superficial de Latossolo vermelho, cultivado com pastagem (T3), como mostra a Figura 2.

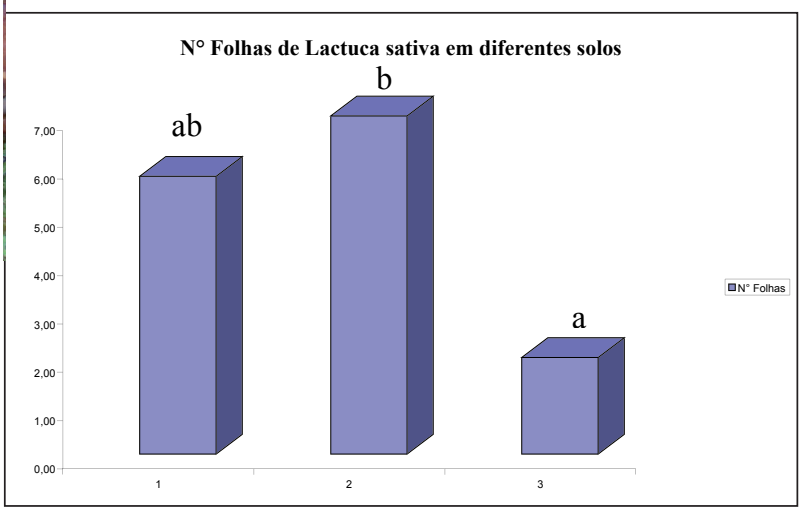

FIGURA 2 - Número de folhas de alface cultivada em diferentes solos.Bambuí, 2009

A alelopatia pode ser ocasionada por volatilização de compostos, liberação decompostos pelas folhas, lixiviação, biodegradação de folhas no solo por microorganismos e exudação das raízes. Segundo Ferreira e Borghetti (2004), esta interferência sobre o desenvolvimento de outra planta pode ser indireta, por meio de transformação destas substâncias no solo pela atividade de microorganismos.

O E. camaldulensis é uma espécie que possui alelopatia sobre algumas culturas, interferindo no seu desenvolvimento, assim como outras espécies florestais. Souza et. al (2007), ao analisarem o comportamento da alface germinada em diferentes concentrações do extrato aquoso de Aroeira (Schinus terebinthifolius), observaram que houve um efeito alelopático desta espécie sobre a germinação da alface na concentração de 100\% do extrato. Indicando a possível presença de um composto inibidor do desenvolvimento desta cultura em áreas anteriormente cultivadas com aroeira.

Rezende et. al (2003), ao estudarem o efeito da incorporação de folhas ou raízes em solo de Eucalyptus sp. sobre o desenvolvimento de leguminosas forrageiras, concluíram que no solo de Itapetininga, explorado anteriormente com eucalipto, havia efeito inibidor ao cultivo das forrageiras, ao passo que esse fato não ocorreu no solo de Nova Odessa, onde anteriormente não se cultivou eucalipto.

Devido a isso, pode-se observar que o solo superficial de áreas com este cultivo, neste caso não exerceu influência sobre o desenvolvimento de alface, pelo contrário, o solo cultivado com esta espécie apresentou o melhor desenvolvimento, com médias de produção superiores aos demais tratamentos.

Segundo Almeida (1991), estes compostos liberados pelas plantas no meio ambiente podem agir de maneira benéfica ou prejudicial. Neste caso, provavelmente não houve interferência alelopática do $E$. camaldulensis, provavelmente o solo coletado sob a sua copa não havia a presença de compostos prejudiciais ao desenvolvimento da cultura. 
Já o solo superficial de um Latossolo Vermelho cultivado com pastagem apresentou um desenvolvimento muito inferior quando comparado às médias dos demais tratamentos. Como o solo estava sob cultivo de pastagem pode ser que a presença de alguma planta daninha presente na área, ou mesmo a própria Brachiaria $s p$. possa ter interferido no desenvolvimento da alface.

Almeida et al. (1997) observaram que em casa de vegetação a produção de matéria seca da parte aérea e das raízes de centrosema, macrotiloma e estilosantes foi reduzida, quando irrigadas com os extratos aquosos de Brachiaria decumbens, B. humidicola e B. brizantha cv. Marandu e que as espécies de braquiárias estudadas apresentaram elevado potencial alelopático, variando de acordo com a espécie de leguminosa estudada.

Portanto, a espécie de Brachiaria sp. presente sobre este Latossolo Vermelho pode ter exercido influências sobre o desenvolvimento da alface, além deste fator, observou-se também que tal solo possui um alto teor de argila, sendo de fácil compactação, o que pode estar também associado ao retardado da germinação e o crescimento da espécie cultivada.

Segundo Eavis (1972), num solo compactado, a taxa de difusão de oxigênio pode limitar o crescimento radicular em potenciais menores ou iguais à "capacidade de campo". Stone et al (2006) também destaca que a excessiva resistência à penetração pode limitar o crescimento das raízes sob condições mais secas.

\section{CONCLUSÃO}

Os tratamentos apresentaram diferenças estatisticamente signifitivas entre si, porém a alface apresentou melhor desenvolvimento nos tratamento com solo superficial de eucalyptus camaldulensis e eucalyptus urophilla, que não apresentaram efeito alelopático na cultura, porém no tratamento com solo superficial de um Latossolo Vermelho cultivado com pastagem, a cultura apresentou um desenvolvimento muito inferior quando comparado às médias dos demais tratamentos, possivelmente devido à presença de alguma planta daninha presente na área, ou mesmo efeito advindo da própria Brachiaria $s p$. que possa ter interferido no desenvolvimento da alface.

\section{REFERÊNCIAS BIBLIOGRAFICAS}

ALMEIDA, A. R. P.; LUCCHESI, A. A.; ABBADO, M. R. Efeito alelopático de espécies de Brachiaria Griseb. sobre algumas leguminosas forrageiras tropicais. II. Avaliações em casa de vegetação. Boletim da Indústria Animal, Nova Odessa, v. 54, n. 2, p. 55-64, 1997.

ALMEIDA, F. S. Efeitos alelopáticos de resíduos vegetais. Pesquisa Agropecuária Brasileira, Brasília, v. 26, n. 2, p. 221-236, 1991.

EAVIS, B. W. Soil physical condition affecting seedling root growth. I. Mechanical impedance, aeration and moisture availability and moisture levels in a sandy loam soil. Plant and Soil, The Hague, v. 36, n. 3, p. 613-622, 1972.

FERREIRA,A.G.;BORGHETTI,F.Germinação: Do básico ao aplicado. Porto Alegre: Editora Artmed, 2004.

FERREIRA, D.F. SISVAR Versão 5.0. Departamento de Ciências Exatas. UFLA, Lavras, MG, 2007.

FIGUEIRA, F. A. R. Manual de olericultura: cultura e comercialização de hortaliças. 2 ed. São Paulo: Agronômica Ceres, v. 2,1982.

MOLISCH, H. Der Einfluss einer Planze auf die andere Alelophathie. Verlag, Jena: Gustav Fisher, 1937. 106 p.

REZENDE, C.P.; PINTO, J.C.; EVANGELISTA, 
A.R.; SANTOS, I.P.A. Alelopatia e suas interações na formação e manejo de pastagem. Boletim Agropecuário, n. 54, Lavras: Universidade Federal de Lavras, mai. 2003. 55 p.

RICE, E. L. Allelopathy. 2. ed. New York: Academic, 1984. 422 p.

SEVERINO, L. S.; LIMA, R. L. S.; ALBUQUERQUE, R. C.;BELTRÃO, E. M. Alelopatia de plantas daninhas sobre a mamomeira. Embrapa Algodão. $2^{\circ}$ Congresso Brasileiro de Mamona. Anais..., 2007.

SOUZA, C.S.M.; SILVA, W.L.P.; GUERRA, A.M.N.M.; CARDOSO, M.C.R.; TORRES, S.B. Alelopatia do extrato aquoso de folhas de aroeira na germinação de sementes de alface. Revista Verde, v.2, n.2, p.96 - 100, Mossoró, RN, jul./ dez. de 2007

SOUZA, SÉRGIO ALESSANDRO MACHADO. Biotestes na Avaliação da Fitotoxicidade de Extratos Aquosos de Plantas Medicinais Nativas do Rio Grande do Sul. Monografia de conclusão de curso. Universidade Federal de Pelotas - RS, 2005.

STONE, L.F.; SILVEIRA, P.M.; MOREIRA, J.A.A. Atributos Físico-hidricos do solo sob plantio direto. Documentos 191, Santo Antônio de Goiás: Embrapa Arroz e Feijão, GO, Nov. 2006. 39 p. 